\title{
Erupted Odontoma: A Report of Three Cases and A Review of the Literature
}

\author{
Umut Pamukçu(0000-0001-8356-8344) ${ }^{\alpha}$,Nuray Bağcı(0000-0001-9362-723X) ${ }^{\alpha}$, Illkay Peker(0000-0002-2888-2979) ${ }^{\alpha}$
}

Selcuk Dent J, 2021; 8: 926-935 (Doi: 10.15311/selcukdentj.812603)

Basvuru Tarihi: 19 Ekim 2020 Yayına Kabul Tarihi: 13 Subat 2021

\begin{abstract}
Erupted Odontoma: A Report of Three Cases and A Review of the Literature

Odontomas are most common benign odontogenic tumors composed of enamel, dentine, cement, and pulp tissue. The eruption of an odontoma is extremely rare. It may be associated with some complications such as pain, inflammation of the adjacent soft tissues and delayed eruption of permanent teeth. In this report, clinical and radiographic findings of three erupted odontoma cases and a review of the literature are presented. Three of our patients were male and the oldest was 80 years old. Two of the patients were symptomatic and one was asymptomatic. Panoramic radiographs and cone-beam computed tomography images were obtained. In the literature review, we reached 66 papers published between 1989 and 2020 , with a total of 74 cases presented. An erupted odontoma may be associated with pain, inflammation of the adjacent soft tissues, and delayed eruption of permanent teeth. Dentists should pay attention to erupted odontoma because of these serious complications.
\end{abstract}

\section{KEYWORDS}

Odontoma, Erupted odontoma, Odontogenic tumor, Review

The term 'odontoma' was first used in 1866 by Broca who defined it as a tumor formed by the overgrowth of complete dental tissue and in 1946 it was classified by Thoma and Goldman. ${ }^{1}$ Odontomas, which are considered developmental anomalies (hamartomas) rather than true neoplasms are the most common benign odontogenic tumors and composed of enamel, dentin, cementum, and pulp. Various theories have been suggested for etiology of odontomas: local trauma in primary dentition duration, infectious and/or inflammatory processes, hereditary abnormalities, and any alteration in genetic structures that can affect the tooth development. ${ }^{2}$ Odontomas are the most frequently observed, non-cystic, non-aggressive, usually asymptomatic, odontogenic benign lesions, and among the odontogenic tumors their incidence ranges between 22-67\%. ${ }^{3}$ According to the World Health Organization (WHO) classification in 2017, based upon its radiographic, histopathologic, and clinical features, there are two types of odontoma: complex odontoma (CxOD) and compound odontoma (CpOD). ${ }^{4}$ CpOD present organized dental tissues and might contain tooth-like structures that are named denticles. On the contrary, CxOD is consists of all dental tissues that arrange randomly. ${ }^{5}$ Their detection is usually in the early years of life, between the ages of 6 and 46, and the mean is 23

\section{Öz}

Sürmüş Odontoma: Üç Vaka Raporu Ve Literatür Derlemesi

Odontomlar, mine, dentin, sement ve pulpa dokusundan oluşan en yaygın benign odontojenik tümörlerdir. Odontomanın sürmesi oldukça nadirdir. Ağrı, çevredeki yumuşak dokuların iltihaplanması ve kalıcı dişlerin gecikmiş sürmesi gibi bazı komplikasyonlarla ilişkili olabilir. Bu raporda, sürmüş üç odontoma vakasının klinik ve radyografik bulguları ve literatür derlemesi sunulmuştur. Hastalarımızın üçü de erkekti ve en yaşlısı 80 yaşındaydı. Hastaların ikisi semptomatik, biri asemptomatikti. Radyografik incelemede, panoramik radyografiye ilaveten, detaylı bir inceleme yapmak adına konik ışınlı bilgisayarlı tomografi görüntüleri de elde edildi. Literatür taramasında 1989 ve 2020 yılları arasında yayınlanmış ve toplam 74 vakanın sunulduğu 66 yayına ulaştık. Sürmüş bir odontoma, ağrı, bitişik yumuşak dokuların iltihabı ve kalıcı dişlerin sürmesinde gecikme ile ilişkili olabilir. Diş hekimleri, bu ciddi komplikasyonlar nedeniyle sürmüş odontomaya dikkat etmelidir.

\section{ANAHTAR KELIMELER}

Odontoma, Sürmüş odontoma, Odontojenik tümör, Derleme

years old. ${ }^{4}$ The sex predilection is controversial; in some studies CpOD reported more common in males, and CxOD slightly more common in females. ${ }^{5}$ Dilated odontoma represented as a dilatation of the crown and root that cause of a deep enamel-lined invagination and it is a very rare developmental abnormality. ${ }^{6}$ Although it has been described as another type of odontoma by some authors, it was not described as a private asset in the current of odontogenic tumors classification.

Junquera et al. ${ }^{7}$ 'clinically' characterized three types of odontomas: 1) intra-osseous (central) odontoma, 2) extraosseous (peripheral) odontoma occurring only in the soft tissue and 3) erupted odontoma as a result of an intra-osseous odontoma eruption into the oral cavity because of its location coronally to an impacted/erupting tooth or superficially in the bone. The eruption of odontoma in the oral cavity is extremely rare, the first case was reported by Rumel et al. ${ }^{8}$ in 1980 . Amado et al. ${ }^{3}$ reported in a review that eruption occurred in only $1.6 \%$ of all odontomas

\footnotetext{
${ }^{\alpha}$ Gazi University Faculty of Dentistry, Department of Dentomaxillofacial Radiology, Ankara, Turkey
} 
cases. An erupted odontoma may be associated with some complications such as pain, inflammation of the adjacent soft tissues, suppuration associated with infection and delayed eruption of permanent teeth.9

The present study identified three patients with erupted odontoma and offers a literature review in terms of the principal characteristics of these lesions.

\section{CASE REPORTS}

\section{Case 1}

A 21-year-old male reported pain after meals for the last three days and slight swelling on the soft tissues of left the mandible for the last one year. The pain was dull, intermittent, exacerbated by chewing and relieved with medication. No relevant medical or family history was reported.

On extraoral examination, a slight swelling was observed on the left cheek, in the region of mandibular angle (Figure 1a). Intraoral examination revealed an ulcerated zone in the left mandible molar region and a partially erupted hard and dental tissue-like mass on the alveolar ridge (Figure 1b). On palpation, the mass had a bone-like tenderness, and swelling was observed in the affected buccal region that accompanied a shallowing vestibular sulcus. Panoramic radiography revealed a well-defined, non-homogeneous radiopaque mass with irregular borders situated in the left mandible body. The mass extended antero-posteriorly from the distal surface of the second premolar to the anterior border of the mandible ramus and supero-inferiorly from the alveolar crest to the lower third of the mandible body (Figure 1c). The axial and coronal cone-beam computed tomography (CBCT) sections showed an irregular radiopacity surrounded by a radiolucent halo above the crown of impacted molar orienting toward the mandible basis (Figure 1d, 1e). The cortical bone perforation was observed at the top of alveolar crest slightly to lingual aspect. The mass was associated with the mandibular canal and caused expansion in both buccal and lingual directions. The approximate size of the mass was $2.9 \mathrm{~cm} \times 1.4 \mathrm{~cm} \times 1.8 \mathrm{~cm}$ (dimensions of anteroposterior $\times$ buccolingual $\times$ superoinferior). The impacted tooth was in close neighborhood of the mandibular canal and caused perforation in the buccal cortical bone (Figure 1f, 1g). The evidence of another molar was not noted, and its absence information could not be obtained from the patient.

Based on the clinical and radiological features, the lesion was pre-diagnosed as CxOD. After the acute symptoms were treated by medication, the patient was referred to the Department of Oral and Maxillofacial Surgery for excision of the lesion. Initially, an incisional biopsy was performed; however, the material obtained was insufficient for histopathological examination. A new operation was carried out for an excisional biopsy under general anesthesia. Histopathological evaluation of the material revealed that it was compatible with CxOD. The lesion was diagnosed as erupted CxOD.

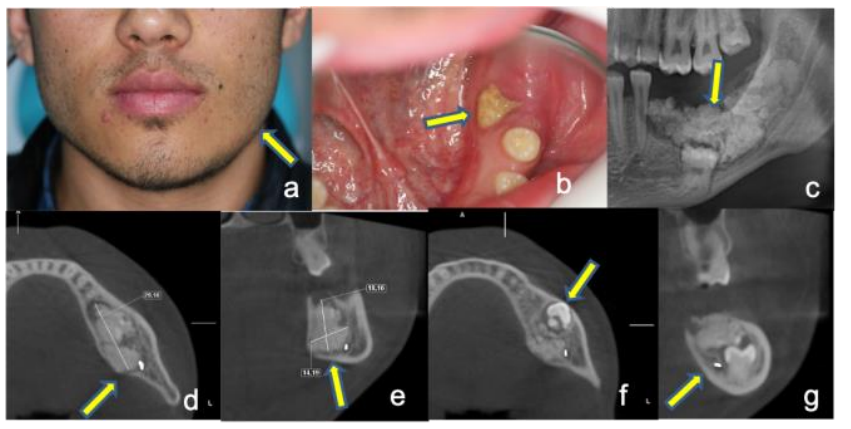

Figure 1

Extraoral view of the swelling of the cheek (arrow) (a), intraoral view of the erupted lesion (arrow) (b), localization of the lesion and impacted tooth on the cropped panoramic radiograph (arrow) (c), axial CBCT section showing the lesion (arrow) (d), coronal CBCT section showing the lesion (arrow) (e), axial CBCT section showing the tooth (f), coronal CBCT section showing the tooth (arrow) (g)

\section{Case 2}

A 23-year-old male was referred to our department by a dentist because of a lesion located in his left mandible. According to the patient's statement, swelling and purulent exudate started in the left posterior region of his mandible five months ago. No relevant medical or family history was reported.

There were no extraoral findings. On intraoral examination, a partially erupted, yellowish-brown, irregularly shaped solid mass was observed on the left posterior mandibular alveolar ridge. The mandibular left third molar was not observed in the oral cavity (Figure 2a). The mass and the related area were tender on palpation. Panoramic radiography revealed a unilateral, non-homogeneous radiopacity above the impacted left third molar crown, at the distal surface of the second molar and associated with its crown and root (Figure $2 \mathrm{~b}$ ). It also perforated the alveolar crest and extending to the oral cavity. The axial and coronal CBCT sections provided a more detailed view of the lesion. The cortical bones at the top of the alveolar crest and additionally at the lingual and buccal sides were perforated (Figure 2d, $2 e)$. The dimensions of the irregular radiopacity that surrounded with a radiolucent rim were $1.3 \mathrm{~cm} \times 1.5$ $\mathrm{cm} \times 1.8 \mathrm{~cm}$ (dimensions of anteroposterior $\times$ buccolingual $\times$ superoinferior). The roots of impacted third molar were towards the basis of the mandible and were in a relationship with the mandibular canal. The impacted tooth was in contact with the mandibular canal and perforated the buccal cortical bone (Figure 2f, 2g)

Based on the clinical and radiological features, the lesion was pre-diagnosed as CxOD. After the acute symptoms were treated by medication, the patient was referred to the Department of Oral and Maxillofacial Surgery for excision of the lesion and the mass was removed (Figure 2c). Histopathological evaluation of the material obtained after the surgery was compatible with CxOD. The lesion was diagnosed as erupted CxOD. 


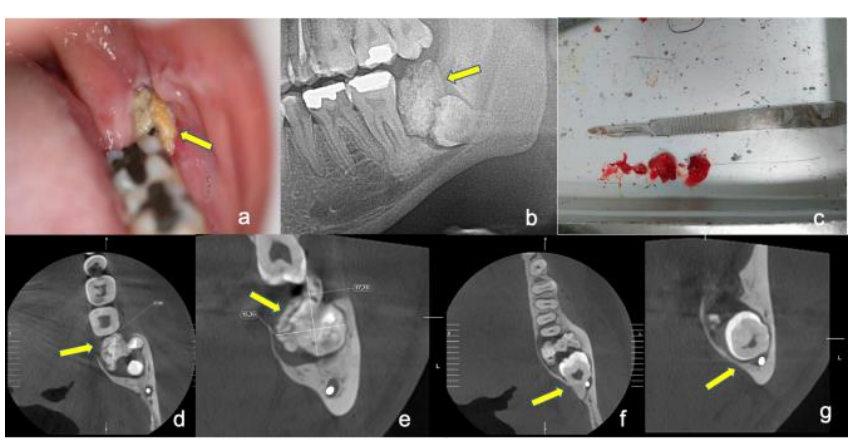

Figure 2

Intraoral view of the erupted lesion (arrow) (a), localization of the lesion and impacted tooth on the cropped panoramic radiograph (arrow) (b), macroscopic view of the specimen and impacted tooth (c), axial CBCT section showing the lesion (arrow) (d), coronal CBCT section showing the lesion (arrow) (e), axial CBCT section showing the impacted tooth (arrow) (f), coronal CBCT section showing the impacted tooth (arrow) (g)

Case 3

An 80-year-old male was referred to our department by a dentist because of a bone-hard mass in his maxilla. The primary expectation of the patient was to replace the old upper and lower partial dentures due to their non-adaptation. He also reported that a tooth/bone-hard mass appeared five years ago in the right posterior maxilla region, but it has been asymptomatic so far.

There was no abnormality on extraoral examination. Intraoral examination revealed a partially erupted yellowish hard mass with an irregular surface on the edentulous right tuber region (Figure 3a). On palpation, the mass was bone-hard. Panoramic radiography revealed a unilateral and nonhomogeneous radiopacity surrounded by a radiolucent rim in the right tuber region (Figure $3 b$ ). The axial and coronal CBCT sections showed perforation in the cortical bone at the top and buccal aspect of the alveolar crest and additionally the mass was in a relationship with maxillary sinus due to perforation on its inferior wall (Figure $3 d, 3 e$ ). The size of the lesion was approximately $1.6 \mathrm{~cm} \times 1.2 \mathrm{~cm} \times$ $1.7 \mathrm{~cm}$ (dimensions of anteroposterior $\times$ buccolingual $\times$ superoinferior). The surgical operation was performed intraorally under local anesthesia and the mass was removed (Figure $3 \mathrm{c}$ ). The histopathological analysis confirmed the diagnosis of CxOD that showed an irregular arrangement of dental tissues such as enamel, dentine, and cementum. The lesion was diagnosed as erupted CxOD.

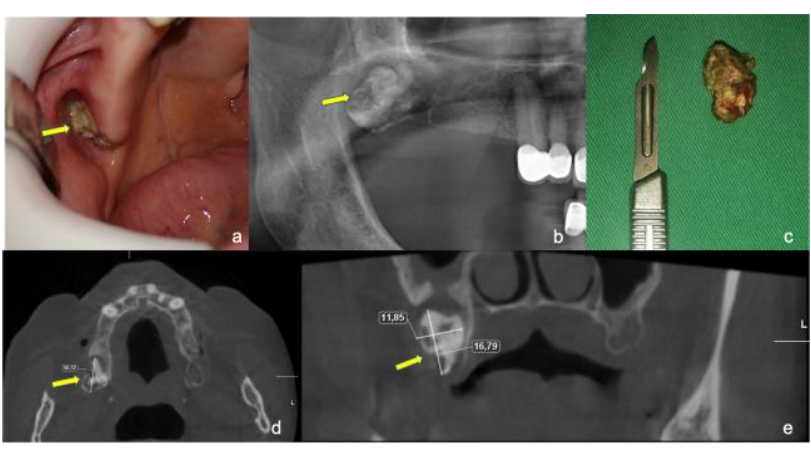

Figure 3

Intraoral view of the erupted lesion (arrow) (a), localization of the lesion and impacted tooth on the cropped panoramic radiograph (arrow) (b), macroscopic view of the specimen (c), axial CBCT section showing the lesion (arrow) (d), coronal CBCT section showing the perforation on the maxillary sinus floor and the lesion (arrow) (e)

\section{LITERATURE RESEARCH}

We conducted a literature search at PubMed and Google Scholar using the keywords "odontoma", "erupted odontoma" and "odontogenic tumor". No restrictions were used regarding the language of the papers. The papers published in a foreign language were translated. This research yielded 66 papers relevant erupted odontoma cases between 1989 and 2020. Totally 74 erupted odontoma cases were reported in 73 patients (one patient had bilateral erupted dilated odontomas (Mahmoodi A et al. ${ }^{10}$ )) (Table 1). The ages of 72 patients ranged between 2 and 68, and the mean age was 20.7. In a paper, two cases that belong to two different patients have been presented; however, the age of one patient was not specified (Bhattacharya PT et al. ${ }^{11}$ ). Regarding the sex, $53.4 \%(n=39)$ of the patients were female and $46.6 \%$ $(n=34)$ were male, that's why we can say the erupted odontoma has no sex predilection. The distribution of jaw involvement was as $47.3 \%(n=35)$ in mandible and $52.7 \%(n=39)$ in maxilla. Among all cases that we have reviewed, 59.4\% $(n=44)$ cases were symptomatic and $40.6 \%(n=30)$ were asymptomatic (Table 2). In the reports stated the situation, $74.3 \%$ $(n=55)$ of the erupted odontoma cases were associated with an impacted tooth. Size was stated for only 45 cases, and the size of these cases ranged from 0.1 to $10 \mathrm{~cm}$. Nineteen (42.2\%) of them were three $\mathrm{cm}$ or more in size and could called a giant odontomas. Except one (Sarojini BS et al.12), there were 73 cases in which the type was specified. The distribution was as $65.8 \% \quad(n=48)$ CxOD, $30.1 \%$ $(n=22)$ CpOD, and $4.1 \%(n=3)$ dilated odontomas. We have not come across a report that reveals a radiographically different situation other than the classical appearance of an odontoma. 
Table 1.

\section{Analysis of erupted odontoma cases reported in the literature}

\begin{tabular}{|c|c|c|c|c|c|c|c|c|}
\hline Author & $\begin{array}{l}\text { Number of } \\
\text { cases }\end{array}$ & Age & Gender & $\begin{array}{l}\text { Type of } \\
\text { odontoma }\end{array}$ & Location & Size & Symptom & $\begin{array}{l}\text { Impacted } \\
\text { tooth }\end{array}$ \\
\hline 1980 Rumel A et al. ${ }^{8}$ & 1 & 17 & Female & Complex & Posterior mandible & - & + & Yes \\
\hline 1980 Birnbaum M et al. ${ }^{13}$ & 1 & 68 & Female & Complex & Anterior maxilla & $1 \mathrm{~cm}$ & - & No \\
\hline 1985 Al-sahhar WF-Putrus ST ${ }^{14}$ & 1 & 12 & Female & Complex & Anterior maxilla & - & + & Yes \\
\hline 1987 Serio FG-Levy BA ${ }^{15}$ & 1 & 41 & Male & Compound & Posterior mandible & - & + & No \\
\hline 1989 Gomel M-Seçkin $T^{16}$ & 1 & 16 & Female & Complex & $\begin{array}{l}\text { Right posterior } \\
\text { maxilla }\end{array}$ & $2 \mathrm{~cm}$ & + & Yes \\
\hline 1992 Lopez-Areal L et al. ${ }^{17}$ & 1 & 12 & Female & Compound & Anterior maxilla & - & - & Yes \\
\hline 1992 White CS-Rogers $J^{18}$ & 1 & 32 & Female & Compound & Anterior maxilla & - & + & No \\
\hline 1993 Nik-Hussein N-Majid Z ${ }^{19}$ & 1 & 8 & Female & Compound & Anterior maxilla & - & - & Yes \\
\hline 1998 Kaneko M et al. ${ }^{20}$ & 1 & 14 & Female & Complex & $\begin{array}{l}\text { Right posterior } \\
\text { mandible }\end{array}$ & $1.5 \times 1 \mathrm{~cm}$ & - & Yes \\
\hline 1999 Botha $\mathrm{PJ}^{21}$ & 1 & 27 & Female & Compound & Anterior mandible & - & - & No \\
\hline 2000 Ragalli CC et al. ${ }^{2}$ & 1 & 59 & Male & Complex & $\begin{array}{l}\text { Right posterior } \\
\text { maxilla }\end{array}$ & $3.5 \mathrm{~cm}$ & - & No \\
\hline 2001 Köymen R et al. ${ }^{22}$ & 1 & 20 & Male & Complex & $\begin{array}{l}\text { Right posterior } \\
\text { mandible }\end{array}$ & $2 \times 2 \mathrm{~cm}$ & + & Yes \\
\hline 2001 Ferrer MR et al. ${ }^{23}$ & 1 & 22 & Female & Complex & $\begin{array}{l}\text { Left posterior } \\
\text { maxilla }\end{array}$ & - & + & Yes \\
\hline 2003 Amado SC et al. ${ }^{3}$ & 1 & 26 & Male & Complex & $\begin{array}{l}\text { Left posterior } \\
\text { maxilla }\end{array}$ & $1 \mathrm{~cm}$ & - & Yes \\
\hline 2004 Litonjua L et al. ${ }^{24}$ & 1 & 17 & Female & Complex & $\begin{array}{l}\text { Left posterior } \\
\text { mandible }\end{array}$ & - & + & Yes \\
\hline 2005 Junquera L et al. ${ }^{7}$ & 1 & 23 & Male & Complex & $\begin{array}{l}\text { Left posterior } \\
\text { maxilla }\end{array}$ & $3 \mathrm{~cm}$ & - & Yes \\
\hline 2007 Vengal M et al. ${ }^{25}$ & 1 & 23 & Male & Complex & $\begin{array}{l}\text { Right posterior } \\
\text { mandible }\end{array}$ & $3.5 \times 3.3 \mathrm{~cm}$ & + & Yes \\
\hline 2007 Bertolai $\mathrm{R}$ et al. ${ }^{26}$ & 1 & 29 & Female & Complex & Posterior mandible & - & + & No \\
\hline 2008 Amailuk P-Grubor D 27 & 1 & 15 & Male & Compound & Anterior maxilla & $2 \mathrm{~cm}$ & - & No \\
\hline \multirow{2}{*}{2008 Ilief-Ala MA et al. ${ }^{28}$} & \multirow{2}{*}{1} & 2 & Female & Complex & Posterior maxilla & - & + & Yes \\
\hline & & 11 & Female & Complex & $\begin{array}{l}\text { Right posterior } \\
\text { mandible }\end{array}$ & $2 \mathrm{~cm}$ & + & Yes \\
\hline \multirow{2}{*}{2009 Serra-Serra G et al.9 } & \multirow{2}{*}{3} & 26 & Male & Complex & $\begin{array}{l}\text { Left posterior } \\
\text { maxilla }\end{array}$ & $1 \mathrm{~cm}$ & - & No \\
\hline & & 27 & Male & Compound & Anterior mandible & - & - & Yes \\
\hline 2009 Shekar S et al. ${ }^{29}$ & 1 & 15 & Female & Compound & $\begin{array}{l}\text { Right posterior } \\
\text { mandible }\end{array}$ & $0.6 \times 0.8 \mathrm{~cm}$ & - & No \\
\hline 2009 Vasudevan V et al. ${ }^{30}$ & 1 & 19 & Female & Complex & $\begin{array}{l}\text { Right posterior } \\
\text { mandible }\end{array}$ & $3 \times 3.5$ & + & No \\
\hline 2010 Chandra S et al. ${ }^{31}$ & 1 & 21 & Female & Compound & $\begin{array}{l}\text { Right posterior } \\
\text { mandible }\end{array}$ & $0.9 \times 0.7 \mathrm{~cm}$ & - & No \\
\hline 2010 Yildirim U $\mathrm{U}^{32}$ & 1 & 22 & Female & Complex & $\begin{array}{l}\text { Right posterior } \\
\text { maxilla }\end{array}$ & $2 \mathrm{~cm}$ & - & Yes \\
\hline 2010 Kodali RM et al. ${ }^{33}$ & 1 & 17 & Male & Complex & $\begin{array}{l}\text { Right posterior } \\
\text { mandible }\end{array}$ & - & + & Yes \\
\hline 2010 Biocic J et al. ${ }^{34}$ & 1 & 10 & Female & Complex & $\begin{array}{l}\text { Right posterior } \\
\text { mandible }\end{array}$ & $5 \times 3 \mathrm{~cm}$ & + & Yes \\
\hline 2011 Avinash Tejasvi $\mathrm{M}^{35}$ & 1 & 22 & Female & Compound & Anterior mandible & $0.5 \mathrm{~cm}$ & - & No \\
\hline 2011 Nisha D et al. ${ }^{36}$ & 1 & 25 & Male & Complex & $\begin{array}{l}\text { Right posterior } \\
\text { mandible }\end{array}$ & $3.5 \mathrm{~cm}$ & + & Yes \\
\hline 2011 Wanjari $P$ et al. ${ }^{37}$ & 1 & 15 & Male & Complex & $\begin{array}{l}\text { Right posterior } \\
\text { maxilla }\end{array}$ & - & - & Yes \\
\hline 2011 Carvalho CHPd et al. ${ }^{38}$ & 1 & 25 & Male & Complex & $\begin{array}{l}\text { Left posterior } \\
\text { maxilla }\end{array}$ & $7 \mathrm{~cm}$ & - & Yes \\
\hline 2012 Arunkumar K-Vijaykumar NG ${ }^{39}$ & 1 & 22 & Male & Complex & $\begin{array}{l}\text { Left posterior } \\
\text { maxilla }\end{array}$ & $4 \times 3.5 \mathrm{~cm}$ & + & Yes \\
\hline 2012 Patil S et al. ${ }^{40}$ & 2 & 25 & Male & Complex & $\begin{array}{l}\text { Left posterior } \\
\text { maxilla }\end{array}$ & $0.5 \mathrm{~cm}$ & + & Yes \\
\hline \multirow{2}{*}{2012 Kaur GA et al. ${ }^{41}$} & & 22 & Male & Complex & $\begin{array}{l}\text { Right posterior } \\
\text { mandible }\end{array}$ & $0.4 \mathrm{~cm}$ & + & Yes \\
\hline & 1 & 23 & Male & Complex & $\begin{array}{l}\text { Right posterior } \\
\text { mandible }\end{array}$ & $3 \times 3 \mathrm{~cm}$ & + & Yes \\
\hline
\end{tabular}




\begin{tabular}{|c|c|c|c|c|c|c|c|c|}
\hline 2012 Hegde S-Pal S ${ }^{42}$ & 1 & 27 & Male & Compound & $\begin{array}{l}\text { Left posterior } \\
\text { maxilla }\end{array}$ & $1.5 \times 1 \mathrm{~cm}$ & + & Yes \\
\hline 2012 Mahmoodi A et al. ${ }^{10}$ & 2 & 22 & Female & Bilateral dilated & $\begin{array}{l}\text { Right-left anterior } \\
\text { maxilla }\end{array}$ & - & + & Yes \\
\hline 2013 Ali Azhar D et al. ${ }^{43}$ & 1 & 17 & Male & Complex & $\begin{array}{l}\text { Left posterior } \\
\text { maxilla }\end{array}$ & - & + & Yes \\
\hline 2013 Padmanabhan M et al. ${ }^{44}$ & 1 & 12 & Male & Complex & $\begin{array}{l}\text { Left posterior } \\
\text { mandible }\end{array}$ & $0.6 \times 1.3 \mathrm{~cm}$ & + & Yes \\
\hline 2013 Mehta D et al. ${ }^{45}$ & 1 & 15 & Female & Compound & Anterior maxilla & - & - & No \\
\hline 2013 Ohtawa Y et al. ${ }^{46}$ & 1 & 10 & Female & Complex & $\begin{array}{l}\text { Right posterior } \\
\text { maxilla }\end{array}$ & $1 \mathrm{~cm}$ & - & Yes \\
\hline \multirow{2}{*}{2014 Sarojini BS et al. ${ }^{12}$} & \multirow{2}{*}{2} & 12 & Male & Compound & Anterior maxilla & - & - & No \\
\hline & & 16 & Female & - & Anterior maxilla & - & - & Yes \\
\hline 2014 Raval N et al. ${ }^{47}$ & 1 & 22 & Male & Compound & Anterior maxilla & - & - & Yes \\
\hline 2014 Murphy C et al. ${ }^{48}$ & 1 & 13 & Male & Complex & $\begin{array}{l}\text { Right posterior } \\
\text { maxilla }\end{array}$ & - & + & No \\
\hline \multirow{2}{*}{2014 Lone PA et al. ${ }^{49}$} & \multirow{2}{*}{2} & 15 & Male & Complex & $\begin{array}{l}\text { Left posterior } \\
\text { mandible }\end{array}$ & $3.5 \mathrm{~cm}$ & + & Yes \\
\hline & & 40 & Male & Complex & $\begin{array}{l}\text { Right posterior } \\
\text { mandible }\end{array}$ & $2 \times 1 \mathrm{~cm}$ & + & Yes \\
\hline \multirow{2}{*}{2015 Özcan G et al. ${ }^{50}$} & \multirow{2}{*}{2} & 14 & Female & Compound & $\begin{array}{l}\text { Left posterior } \\
\text { maxilla }\end{array}$ & - & - & Yes \\
\hline & & 35 & Female & Compound & Anterior mandible & - & - & No \\
\hline 2015 Bagewadi SB et al. ${ }^{51}$ & 1 & 22 & Male & Complex & $\begin{array}{l}\text { Right posterior } \\
\text { mandible }\end{array}$ & $3.5 \times 4 \mathrm{~cm}$ & - & Yes \\
\hline 2015 Bereket $C$ et al. ${ }^{52}$ & 1 & 19 & Male & Compound & $\begin{array}{l}\text { Left posterior } \\
\text { maxilla }\end{array}$ & $0.1 \times 0.1 \mathrm{~cm}$ & + & Yes \\
\hline 2015 Kudva A et al. ${ }^{53}$ & 1 & 23 & Female & Complex & $\begin{array}{l}\text { Right posterior } \\
\text { mandible }\end{array}$ & $3 \times 3.5 \mathrm{~cm}$ & + & Yes \\
\hline 2015 Venigalla A et al. ${ }^{54}$ & 1 & 6 & Female & Compound & $\begin{array}{l}\text { Right posterior } \\
\text { maxilla }\end{array}$ & $1 \times 2 \mathrm{~cm}$ & + & Yes \\
\hline 2015 Almeida LE et al. ${ }^{55}$ & 1 & 17 & Male & Complex & $\begin{array}{l}\text { Right posterior } \\
\text { mandible }\end{array}$ & $2 \mathrm{~cm}$ & + & Yes \\
\hline 2015 Ahmed KA ${ }^{56}$ & 1 & 24 & Male & Complex & $\begin{array}{l}\text { Right posterior } \\
\text { mandible }\end{array}$ & - & + & Yes \\
\hline \multirow{2}{*}{2015 Bhattacharya PT et al. ${ }^{11}$} & \multirow{2}{*}{2} & 30 & Female & Complex & $\begin{array}{l}\text { Right posterior } \\
\text { mandible }\end{array}$ & $3 \times 2 \mathrm{~cm}$ & + & No \\
\hline & & - & Female & Complex & $\begin{array}{l}\text { Right posterior } \\
\text { mandible }\end{array}$ & $4 \times 3 \mathrm{~cm}$ & - & Yes \\
\hline 2016 de Pró Lizuaín C et al. ${ }^{4}$ & 1 & 22 & Female & Complex & $\begin{array}{l}\text { Left posterior } \\
\text { mandible }\end{array}$ & $3.6 \times 3 \mathrm{~cm}$ & + & Yes \\
\hline 2016 Dutta SR et al. ${ }^{57}$ & 1 & 13 & Male & Complex & $\begin{array}{l}\text { Left posterior } \\
\text { maxilla }\end{array}$ & $10 \mathrm{~cm}$ & + & Yes \\
\hline 2016 Sharma G et al. ${ }^{58}$ & 1 & 18 & Male & Dilated & Anterior maxilla & - & + & Yes \\
\hline 2017 Hasanuddin S- Reddy ER ${ }^{59}$ & 1 & 7 & Female & Compound & $\begin{array}{l}\text { Left posterior } \\
\text { mandible }\end{array}$ & - & + & No \\
\hline 2017 Sabir H-Reddy ER ${ }^{60}$ & 1 & 19 & Female & Complex & $\begin{array}{l}\text { Left posterior } \\
\text { mandible }\end{array}$ & $1.4 \times 0.9 \mathrm{~cm}$ & + & Yes \\
\hline 2017 Swaidan $\mathrm{RF}^{61}$ & 1 & 16 & Male & Complex & $\begin{array}{l}\text { Left posterior } \\
\text { maxilla }\end{array}$ & $2 \mathrm{~cm}$ & + & Yes \\
\hline 2017 Deshpande $\mathrm{N}$ et al. ${ }^{62}$ & 1 & 18 & Male & Complex & $\begin{array}{l}\text { Right posterior } \\
\text { maxilla }\end{array}$ & $3 \times 3.5 \mathrm{~cm}$ & + & Yes \\
\hline 2018 Katoumas K et al. ${ }^{63}$ & 1 & 23 & Male & Complex & $\begin{array}{l}\text { Left posterior } \\
\text { mandible }\end{array}$ & $2.5 \times 2 \times 2 \mathrm{~cm}$ & + & Yes \\
\hline 2018 Coşgun A et al. ${ }^{64}$ & 1 & 12 & Female & Complex & $\begin{array}{l}\text { Right posterior } \\
\text { mandible }\end{array}$ & $\begin{array}{c}0.8 \times 0.7 \times 0.7 \\
\mathrm{~cm}\end{array}$ & + & No \\
\hline 2018 Sulabha AN et al. ${ }^{65}$ & 1 & 16 & Female & Compound & $\begin{array}{l}\text { Right anterior } \\
\text { maxilla }\end{array}$ & - & - & Yes \\
\hline 2019 Zhuoying C-Fengguo $\mathrm{Y}^{66}$ & 1 & 14 & Female & Complex & $\begin{array}{l}\text { Left posterior } \\
\text { maxilla }\end{array}$ & $\begin{array}{c}2.6 \times 2.4 \times 4.2 \\
\mathrm{~cm}\end{array}$ & - & Yes \\
\hline 2019 Honnegowda DKK et al. ${ }^{67}$ & 1 & 32 & Female & Compound & $\begin{array}{l}\text { Right anterior } \\
\text { maxilla }\end{array}$ & $1 \times 1.2 \mathrm{~cm}$ & + & Yes \\
\hline 2019 Niazmand M-Mokhtari S $^{68}$ & 1 & 18 & Female & Complex & $\begin{array}{l}\text { Right posterior } \\
\text { mandible }\end{array}$ & $5 \times 1 \mathrm{~cm}$ & - & Yes \\
\hline 2020 Marcarini KNO et al. ${ }^{69}$ & 1 & 24 & Female & Compound & $\begin{array}{l}\text { Right anterior } \\
\text { maxilla }\end{array}$ & - & - & Yes \\
\hline
\end{tabular}


Table 2.

\section{Symptoms of erupted odontoma cases reported in the literature}

\begin{tabular}{|c|c|c|}
\hline Author & $\begin{array}{l}\text { Number of } \\
\text { cases }\end{array}$ & Symptom \\
\hline 1980 Rumel A et al. ${ }^{8}$ & 1 & Pain, infection \\
\hline 1985 Al-sahhar WF-Putrus ST ${ }^{14}$ & 1 & Infection \\
\hline 1987 Serio FG-Levy BA ${ }^{15}$ & 1 & Tongue irritation \\
\hline 1989 Gomel M-Seçkin T ${ }^{16}$ & 1 & Pain, swelling \\
\hline 1992 White CS-Rogers $\mathrm{J}^{18}$ & 1 & Malocclusion \\
\hline 2001 Köymen R et al. ${ }^{22}$ & 1 & Pain, swelling \\
\hline 2001 Ferrer MR et al. ${ }^{23}$ & 1 & $\begin{array}{l}\text { Pain, swelling, } \\
\text { infection }\end{array}$ \\
\hline 2004 Litonjua L et al. ${ }^{24}$ & 1 & Pain, swelling \\
\hline 2007 Vengal $\mathrm{M}$ et al. ${ }^{25}$ & 1 & Pain, swelling \\
\hline 2007 Bertolai $\mathrm{R}$ et al. ${ }^{26}$ & 1 & $\begin{array}{l}\text { Swelling, Ludwig's } \\
\text { angina }\end{array}$ \\
\hline 2008 llief-Ala MA et al. ${ }^{28}$ & 1 & Bleeding \\
\hline 2009 Serra-Serra G et al. ${ }^{9}$ & 1 & Pain, swelling \\
\hline 2009 Vasudevan V et al. ${ }^{30}$ & 1 & Pain, swelling \\
\hline 2010 Kodali RM et al. ${ }^{33}$ & 1 & Pain \\
\hline 2010 Biocic J et al. $^{34}$ & 1 & Pain, swelling \\
\hline 2011 Nisha D et al. ${ }^{36}$ & 1 & Pain \\
\hline 2012 Arunkumar K-Vijaykumar NG ${ }^{39}$ & 1 & Pain, swelling \\
\hline 2012 Patil S et al. ${ }^{40}$ & 2 & Swelling \\
\hline 2012 Kaur GA et al..$^{41}$ & 1 & Pain, swelling \\
\hline 2012 Hegde S-Pal S ${ }^{42}$ & 1 & Pain, swelling \\
\hline 2012 Mahmoodi A et al. ${ }^{10}$ & 2 & Pain, swelling \\
\hline 2013 Ali Azhar D et al. ${ }^{43}$ & 1 & Pain \\
\hline 2013 Padmanabhan M et al. ${ }^{44}$ & 1 & Pain, swelling \\
\hline 2014 Murphy $C$ et al. ${ }^{48}$ & 1 & Infection \\
\hline 2014 Lone PA et al. ${ }^{49}$ & 2 & Pain, swelling \\
\hline 2015 Bereket C et al. ${ }^{52}$ & 1 & Swelling \\
\hline 2015 Kudva A et al. ${ }^{53}$ & 1 & Pain, swelling \\
\hline 2015 Venigalla $A$ et al..$^{54}$ & 1 & $\begin{array}{l}\text { Pain, difficult in } \\
\text { mouth opening }\end{array}$ \\
\hline 2015 Almeida LE et al. ${ }^{55}$ & 1 & Infection \\
\hline 2015 Ahmed KA ${ }^{56}$ & 1 & Infection \\
\hline 2015 Bhattacharya PT et al. ${ }^{11}$ & 1 & Pain \\
\hline 2016 de Pró Lizuaín C et al. ${ }^{4}$ & 1 & Swelling \\
\hline 2016 Dutta SR et al..$^{57}$ & 1 & Pain, swelling \\
\hline 2016 Sharma G et al. ${ }^{58}$ & 1 & Pain \\
\hline 2017 Hasanuddin S- Reddy ER ${ }^{59}$ & 1 & Pain \\
\hline 2017 Sabir H-Reddy ER ${ }^{60}$ & 1 & Pain, swelling \\
\hline 2017 Swaidan $\mathrm{RF}^{61}$ & 1 & Cellulitis \\
\hline 2017 Deshpande $\mathrm{N}$ et al. ${ }^{62}$ & 1 & Swelling \\
\hline 2018 Katoumas K et al. ${ }^{63}$ & 1 & Swelling \\
\hline 2018 Coşgun A et al. ${ }^{64}$ & 1 & Pain \\
\hline 2019 Honnegowda DKK et al. ${ }^{67}$ & 1 & Pain \\
\hline
\end{tabular}

\section{DISCUSSION}

Various theories have been proposed regarding the process erupting of an odontoma into the oral cavity. 9 Considering the eruption of a tooth, the possible mechanism of the eruption of an odontoma is assumed to be the force of the impacted tooth that is located under the odontoma. ${ }^{7,30}$ However, the process seems to be different from tooth eruption due to the lack of the periodontal ligament and root, so it may be inaccurate to say it is an eruption. In cases where the impacted teeth are not observed, the reasons could possibly be the increasing size of odontoma, bone resorption, the sequestration of overlying bone, remodeling of the jaws or reactive growth of the capsule that surrounds the odontoma. ${ }^{2,25}$ The immunohistochemical investigations have suggested that the cellular activity involving the reduced enamel epithelium and the follicle is associated with the eruption. However, a follicle is needed indirectly for the eruption. The follicle provides both the conductance and chemoattraction for osteoclasts that will lead to eruption. ${ }^{53}$ It means, the possible etiology of the eruption is multifactorial, and it has not been so clear until today.

In the present report, ages of the first and second patients were 21 and 23, respectively which overlap with previous reports, but 80 (case 3) was an extremely late age for an erupted odontoma, and no such case has been reported in the literature before. All our three patients were males. In two cases erupted odontoma were localized in mandible and in one was in maxilla. The reason why our findings about sex and jaw involvement were not in accordance with the literature may be the small sample size.

Clinical indicators of odontoma may include the retention of deciduous teeth, the non-eruption of permanent teeth, pain, the expansion of cortical bone and tooth displacement. Although impacted odontoma is usually asymptomatic, as an exceptional case, erupted odontoma has more serious complications like pain, swelling, and inflammation of adjacent soft tissues or suppuration related to infection. ${ }^{7,24}$ Bertolai et al. ${ }^{26}$ reported a case of Ludwig's angina associated with an erupted odontoma. In some cases, facial asymmetry ${ }^{25}$, halitosis ${ }^{16}$, malocclusion ${ }^{17,19}$, and recurrent infection were recorded. ${ }^{14}$ When an odontoma erupts, pain and swelling are the most common symptoms. ${ }^{53}$ In this report, two of the three cases were symptomatic. The first case presented pain and swelling, and the second swelling and purulent exudate. First of all, a symptomatic treatment was applied to our patients who applied to the dentomaxillofacial radiology department with symptoms such as pain, swelling or prulent exudate. After their oral health conditions were stabilized, they were referred to the oral and maxillofacial surgery department. These symptoms were in accordance with the literature. 
Impaction and/or altered eruption of the teeth located under odontoma occur because of the obstruction the eruptive trajectory. ${ }^{2,9,25}$ Of $37-87 \%$ patients with odontoma show delayed eruption of a permanent tooth. ${ }^{46}$ The probability of this phenomenon rises to $86 \%$ in erupted odontomas. ${ }^{46}$ As suggested by Junquera et al. ${ }^{7}$ that most of the erupted odontomas are related to an impacted tooth, the eruptive force of this tooth can play a major role in the eruption of the odontoma. However, Ragalli et al. ${ }^{2}$ suggested that the reactive growth of circumambient capsule of the odontoma may contribute to the eruption. In this report, the first and second cases were associated with an impacted tooth. The eruptive force of these impacted teeth may have played an important role in odontoma eruption. The third case was not associated with an impacted tooth. However, the denture stimulation may have created an eruptive force.

Radiographically odontomas have characteristic features that depend on their developmental stage and the degree of mineralization; the first stage as a radiolucency due to lack of calcification, the intermediate stage as a mixed (radiolucentradiopaque) lesion because of partial calcification, and at the third stage, the lesion usually appears as a radiopaque mass surrounded by a radiolucent rim that histologically corresponds to connective tissue. ${ }^{70}$ In addition, erupted odontomas exhibit perforation on the alveolar cortical bone. The relation with the oral cavity may cause a peripheral radiolucency as a result of possible inflammation. As the surgical extraction was recommended as soon as they were detected for erupted odontomas, CBCT images can be useful in order to determine the certain of their size and localization in the jaw. ${ }^{71}$ So this imaging modality is also valuable for better surgical planning of the impacted lesions because of the reduced risk of damage to surrounding anatomical structures. ${ }^{72}$ In addition, the same applies for impacted teeth associated with odontomas as in our first and second patients. In our three cases, we observed radiopaque masses surrounded by a radiolucent rim compatible with third stage radiographic appearance. Clinical and radiological signs of inflammation in the adjacent tissues were present in the second and third cases.

To prevent cystic degeneration and other acute symptoms, removal of the mass and enveloping soft tissue after the curettage is the common treatment choice. However, there is no general agreement on the best management approach for the impacted tooth associated with odontoma. ${ }^{24,73}$ The treatment options for impacted tooth comprises surgical extraction, fenestration, and posterior orthodontic traction or simple periodic follow-up with clinical and radiological controls to evaluate its course. ${ }^{3,74,75}$ Since treatment results are not widely shared in many cases in the literature, we had to state the general trend. The surgical operation was performed in the present three cases. In the first and second cases, there were impacted teeth, and these were extracted simultaneously with odontomas.

Odontomas have limited growth potential, and the largest dimension they reach is usually up to $3 \mathrm{~cm} .^{76}$ However, in some rare cases, large odontoma51, also known as giant odontoma ${ }^{77}$, which has a diameter exceeding $3 \mathrm{~cm}$, may be observed on the radiograph.76 In a study by Miki et al. ${ }^{78}$, it was reported that only $4.3 \%$ of odontomas were to be larger than 3 $\mathrm{cm}$. Only our first patient had a giant odontoma (33.3\%) and this rate was close to that of the literature.

It was stated that $\mathrm{CpOD}$ and $\mathrm{CxOD}$ were not superior to each other in terms of the incidence of erupted odontomas. ${ }^{9,53}$ All of our three cases were CxOD, and this was in line with the published cases in the literature.

Odontomas can also manifest as a part of some syndromes as Gardner syndrome, Hermann syndrome, familial colonic adenomatosis, and basal cell nevus syndrome. ${ }^{16}$ However, in the literature, there was no report for an erupted odontoma related to a syndrome. In the three present cases we presented, also there was no history a syndrome.

\section{CONCLUSIONS}

We examined three erupted odontoma cases and reviewed published reports about this patholojical entity in the literature. The findings we reached as a result of the literature review showed us these:

1. Erupted odontomas were mostly observed in young patients.

2. They were slightly more common in females.

3. Their localization showed a slight predilection for the maxilla.

4. Clinically, the erupted odontomas were usually symptomatic and the dominant features were pain, swelling and infection.

5. The cases were often associated with an impacted tooth.

6. The treatment for the mass was certainly a surgical approach but there was no consensus for the underlying impacted tooth treatment.

7. A little less than half of the erupted odontomas can be larger than three $\mathrm{cm}$ (giant odontoma).

8. Their histopathologic type was generally diagnosed as CxOD.

The dentists should be aware of clinical features, radiographic manifestations, and treatment options of this benign lesion to avoid undesirable outcomes. The early diagnosis that follows with an appropriate treatment approach could prevent probable complications.

Ethical Approval: The paper described has been carried out in accordance with Declaration of Helsinki. 


\section{KAYNAKLAR}

1. Thoma KH, Goldman HM. Odontogenic tumors: A classification based on observations of the epithelial, mesenchymal, and mixed varieties. Am J Clin Pathol 1946; 22: 433.

2. Ragalli CC, Ferreria JL, Blasco F. Large erupting complex odontoma. Int J Oral Maxillofac Surg 2000; 29: 373-4.

3. Amado SC, Gargallo JA, Berini LA, Gay CE. Review of 61 cases of odontoma. Presentation of an erupted complex odontoma. Med Oral Patol Oral Cir Bucal 2003; 8: 366-73.

4. de Pró Lizuaín C, Fungi Monetti M, Beovide Cortegoso V. Erupted odontoma: A case report and literature review. Odontoestomatologia 2016; 18: 606.

5. Sánchez OH, Berrocal MIL, González JMM. Metaanalysis of the epidemiology and clinical manifestations of odontomas. Med Oral Patol Oral Cir Bucal 2008; 13: E730-4.

6. Matsusue $\mathrm{Y}$, Yamamoto K, Inagake K, Kirita T. A dilated odontoma in the second molar region of the mandible. Open Dent J 2011; 5: 150-3.

7. Junquera L, de Vicente JC, Roig $P$, Olay S, Rodríguez-Recio O. Odontoma intraóseo erupcionado: Una infrecuente patología Intraosseus odontoma erupted into the oral cavity: An unusual pathology. Pathology 2005; 10: 248-51.

8. Rumel A, de Freitas A, Birman EG, Tannous LA, Chacon PT, Borkas S. Erupted complex odontoma. Report of a case. Dentomaxillofac Radiol 1980; 9: 5-9.

9. Serra-Serra G, Berini-Aytes L, Gay-Escoda C. Erupted odontomas: a report of three cases and review of the literature. Med Oral Patol Oral Cir Bucal 2009; 14: E299-303.

10. Mahmoodi A, Shahidi S, Houshyar M, Houshyar M. Bilateral cystic lesions associated to maxillary erupted dilated odontomas: a case report. J Dent (Shiraz) 2012.

11. Bhattacharya PT, Sarkar S, Khaitan T, Kabiraj A. Erupted complex composite odontoma: Report of two atypical cases. J Dent Allied Sci 2015; 4: 99.

12.Bhargavan Sarojini S, Khosla E, Varghese T, Johnson Arakkal L. Eruption of odontomas into the oral cavity: a report of 2 cases. Case Rep Dent 2014; 2014: 639173.

13. Birnbaum M, Tyrangel A, Steinerman SM. Exfoliated odontoma. Oral Surg Oral Med Oral Pathol 1980; 49: 96.

14.Al-Sahhar WF, Putrus ST. Erupted odontoma. Oral Surg Oral Med Oral Pathol 1985; 59: 225-6.
15.Serio FG, Levy BA. Erupted compound odontoma. Review and report of case. Ann Dent 1987; 46: 412, 5.

16. Gomel M, Seckin T. An erupted odontoma: case report. J Oral Maxillofac Surg 1989; 47: 999-1000.

17.Lopez-Areal L, Silvestre Donat F, Gil Lozano J. Compound odontoma erupting in the mouth: 4year follow-up of a clinical case. J Oral Pathol Med 1992; 21: 285-8.

18. White CS, Rogers J. Erupted compound odontoma: review and case report. Gen Dent 1992; 40: 43-4.

19.Nik-Hussein NN, Majid ZA. Erupted compound odontoma. Ann Dent 1993; 52: 9-11.

20.Kaneko M, Fukuda M, Sano T, Ohnishi T, Hosokawa Y. Microradiographic and microscopic investigation of a rare case of complex odontoma. Oral Surg Oral Med Oral Pathol Oral Radiol Endod 1998; 86: 131-4.

21.Botha PJ. An erupted compound odontoma. SADJ 1999; 54: 153.

22. Köymen R, Ortakoğlu K, Aydıntuğ Y, Günaydın Y. An erupted complex odontoma (a case report). Turkiye Klinikleri J Dental Sci 2001; 7: 101-4.

23.Ferrer MR, Silvestre FD, Estelles EF, Grau DGM, López RM. Recurrent infection of a complex odontoma following eruption in the mouth. Med Oral Patol Oral Cir Bucal 2001; 6: 269-75.

24.Litonjua LA, Suresh L, Valderrama LS, Neiders ME. Erupted complex odontoma: a case report and literature review. Gen Dent 2004; 52: 248-51.

25.Vengal M, Arora H, Ghosh S, Pai KM. Large erupting complex odontoma: a case report. J Can Dent Assoc 2007; 73: 169-73.

26. Bertolai R, Acocella A, Sacco R, Agostini $T$. Submandibular cellulitis (Ludwig's angina) associated to a complex odontoma erupted into the oral cavity. Case report and literature review. Minerva Stomatol 2007; 56: 639-47.

27. Amailuk P, Grubor D. Erupted compound odontoma: case report of a 15-year-old Sudanese boy with a history of traditional dental mutilation. Br Dent J 2008; 204: 11-4.

28. Ilief-Ala MA, Eisenberg E, Mathieu G. Peripheral complex odontoma in a pediatric dental patient: a case report. J Mass Dent Soc 2008; 56: 24-6.

29. Shekar S, Rao RS, Gunasheela B, Supriya N. Erupted compound odontome. J Oral Maxillofac Pathol 2009; 13: 47-50.

30.Vasudevan V, Manjunath V, Bavle RM. Large erupted complex odontoma. J Indian Acad Oral Med Radiol 2009; 21: 92-7.

31.Chandra S, Bagewadi A, Keluskar V, Sah K. Compound composite odontome erupting into the oral cavity: A rare entity. Contemp Clin Dent 2010; 1: 123.

32. Yildirim U. An erupted complex odontoma. N Y State Dent J 2010; 76: 52. 
33. Kodali RM, Venkat Suresh B, Ramanjaneya Raju P, Vora SK. An unusual complex odontoma. J Maxillofac Oral Surg 2010; 9: 314-7.

34.Biocic J, Macan D, Brajdic D, Manojlovic S, Butorac-Rakvin L, Hat J. Large erupting complex odontoma in a dentigerous cyst removed by a piecemeal resection. Pediatr Dent 2010; 32: 255-9.

35. Avinash Tejasvi M. Erupted compound odontomas: a case report. J Dent Res Dent Clin Dent Prospects 2011; 5: 33.

36. Nisha D, Rishabh K, Ashwarya T, Sukriti M, Gupta S. An unusual case of erupted composite complex odontoma. J Dent Sci Res 2011; 2: 1-5.

37. Wanjari P, Wanjari S, Bhowate R. Erupting complex odontoma of maxilla: A case report. J J Pierre Fauchard Acad (India Section) 2011; 25: 65-8.

38. Carvalho CHPd, Costa DdA, Queiroz LMG, Amaral JIQd, Germano AR. Extensive complex odontoma in the maxillary sinus: An uncommon presentation as a cause of chronic sinusitis. Revista Odonto Ciência 2011; 26: 92-5.

39. Arunkumar KV, Vijaykumar, Garg N. Surgical management of an erupted complex odontoma occupying maxillary sinus. Ann Maxillofac Surg 2012; 2: 86-9.

40.40. Patil S, Rahman F, Tipu SR, Kaswan S. Erupted odontomas: a report of two unusual cases. RSBO 2012; 9: 199-203.

41. Kaur GA, Sivapathasundharam B, Berkovitz BK, Radhakrishnan RA. An erupted odontoma associated with pigmentation: a histogenetic and histological perspective. Indian J Dent Res 2012; 23: 699.

42. Hegde S, Pal S. Erupted compound odontome associated with impacted maxillary second premolar: a case report. Arch Oral Res 2012; 8.

43. Ali Azhar D, Kota MZ, El-Nagdy S. An unusual erupted complex composite odontoma: a rare case. Case Rep Dent 2013; 2013: 106019.

44.Padmanabhan M, Pandey R, Aparna R. Erupted composite odontoma associated with malformed teeth-unusual dental aberrations following maxillofacial trauma. Rom J Morphol Embryol 2013; 54: 1153-6.

45. Mehta D, Raval N, Udhani S, Parekh V, Modi C. An unusual case report of erupted odontoma. Case Rep Dent 2013; 2013: 570954.

46. Ohtawa Y, Ichinohe S, Kimura E, Hashimoto S. Erupted complex odontoma delayed eruption of permanent molar. Bull Tokyo Dent Coll 2013; 54: 251-7.

47. Raval N, Mehta D, Vachhrajani K, Nimavat A. Erupted odontoma: a case report. J Clin Diagn Res 2014; 8: ZD10-1.

48. Murphy C, O'Connell JE, Cotter E, Kearns G. Management of large erupting complex odontoma in maxilla. Case Rep Pediatr 2014; 2014: 963962.
49.Lone PA, Kour I, Gandral A. Intra oral approach for complex \& compound odontomas (large or small). Modern Plastic Surgery 2014; 2014.

50.Özcan G, Şekerci A, Ekizer A, Kara Ö. Erupted Compound Odontoma: Two Case Reports. Turkiye Klinikleri J Dental Sci Cases 2015; 1: 126-30.

51.Bagewadi SB, Kukreja R, Suma GN, Yadav B, Sharma $H$. Unusually large erupted complex odontoma: A rare case report. Imaging Sci Dent 2015; 45: 49-54.

52. Bereket C, Cakir-Ozkan N, Sener I, Bulut E, Tek M. Complex and compound odontomas: Analysis of 69 cases and a rare case of erupted compound odontoma. Niger J Clin Pract 2015; 18: 726-30.

53.Kudva A, Chithra A, Rao NN, Cariappa KM. An Erupted Silent Tumour. J Maxillofac Oral Surg 2016; 15: 320-4.

54.Venigalla A, Guttikonda LK, Nelakurthi H, Babburi S, Pinisetti $S$, Kotti $A B$, et al. Ectopic compound odontoma in the buccal mucosa: report of a rare case. Case Rep Dent 2015; 2015: 835171.

55. Almeida LE, Andrade $M$, de Oliveira Filho $M$, Trevilatto PC, Doetzer A. Erupted complex odontoma mimicking a mandibular second molar. Int J Dentistry Oral Sci 2015.

56. Ahmed KA. Large eruption complex odontome in a Saudi patient. Saudi Med J 2015; 36: 228-32.

57.Dutta SR, Passi D, Sharma S, Singh P, Ahmed M. Erupted Large Anterior Maxillary Complex Odontoma: A Rare Dental Hamartoma. J Clin Diagn Res 2016; 10: ZJ01-ZJ2.

58.Sharma G, Nagra A, Singh G, Nagpal A, Soin A, Bhardwaj V. An Erupted Dilated Odontoma: A Rare Presentation. Case Rep Dent 2016; 2016: 9750947.

59. Hasanuddin S, Reddy ER. Erupted odontome associated with a primary molar: A unique clinical presentation, 4-year follow-up. J Oral Maxillofac Pathol 2017; 21 : 322.

60. Husain Sabir Mannan M, Subhas Kumbhare M, Rekha Chaudari M, Gupta S. Erupted Complex Odontoma in Unison with Impacted Mandibular Molar Associated with a Dentigerous Cyst. J N J Dent Assoc 2017; 88: 10-3.

61.Swaidan RF. Management of Complex Erupting odontoma in the maxilla. IJDOH 2017; 3: 74-7.

62.Deshpande $N$, Jadhav A, Bhola $N$, et al. Management of an erupting large maxillary complex odontoma - report of an unusual case. IOSR-JDMS 2017; 16: 75-8.

63. Katoumas K, Lianou V, Fyrgiola M, Dimopoulos I, Chrysomali E, Sklavounou A. Large erupted complex odontoma with dentigerous cyst. J Oral Maxillofac Surg Med Pathol 2018; 30: 330-5.

64. Coşgun A, Bolgül B, Meric E, Turgay B. Compound Odontoma-Diagnosis and Treatment in Pediatrıc Dentıstry: Three Case Reports. Clin Surg 2018; 3 : 1971. 
65.Sulabha A, Attar NM, Awati MAM, Suchitra G. Erupted odontoma mimicking peg lateral incisor associated with impacted maxillary lateral incisor and canine-A rare clinical case report. J Med Radiol Pathol Surg 2019; 6: 15-7.

66.Zhuoying C, Fengguo Y. Huge erupted complex odontoma in maxilla. Oral Maxillofac Surg Cases 2019; 5: 100096.

67. Honnegowda DKK, Ranganna VL, Chandregowda KY, Krishna G, Kumar N. Erupted compound odontoma: A rare case report and review. Int J Oral Care Res 2019; 7: 77.

68. Niazmand M, Mokhtari S. Large erupted odontoma of the mandible: Report of a rare case. Res $\mathrm{J}$ Med Sci 2019; 39: 189-.

69. Marcarini KNO, Bessa GDO, Rodrigues BL, Bertollo RM, Venâncio MAAS, Castro MCC, et al. Erupted odontoma associated with impacted teeth-Case report. Oral Surg Oral Med Oral Pathol Oral Radiol 2020; 129: e115.

70. Choudhary PJ, Gharote HP, Hegde K, Gangwal P. Compound odontoma associated with impacted teeth: a case report. IJSS Case Rep Rev 2014; 1: $12-5$.

71.Park JC, Yang JH, Jo SY, Kim BC, Lee J, Lee W. Giant complex odontoma in the posterior mandible: A case report and literature review. Imaging Sci Dent 2018; 48: 289-93.

72.Akay G, Özdede M, Güngör K. Mesiodenslerin Değerlendirilmesi: Konik Işınlı Bilgisayarlı Tomografi ile Retrospektif Bir Çalışma. Selcuk Dent J 2018; 5: 203-11.

73. Hisatomi M, Asaumi Jl, Konouchi H, Honda Y, Wakasa T, Kishi K. A case of complex odontoma associated with an impacted lower deciduous second molar and analysis of the 107 odontomas. Oral Dis 2002; 8: 100-5.

74. Frank CA. Treatment options for impacted teeth. J Am Dent Assoc 2000; 131: 623-32.

75. Liu JK, Hsiao CK, Chen HA, Tsai MY. Orthodontic correction of a mandibular first molar deeply impacted by an odontoma: a case report. Quintessence Int 1997; 28: 381-5.

76.Spini PH, Spini TH, Servato JP, Faria PR, Cardoso SV, Loyola AM. Giant complex odontoma of the anterior mandible: report of case with long follow up. Braz Dent J 2012; 23: 597-600.

77.Perumal CJ, Mohamed A, Singh A, Noffke CE. Sequestrating giant complex odontoma: a case report and review of the literature. J Maxillofac Oral Surg 2013; 12: 480-4.

78. Miki Y, Oda Y, Iwaya N, Hirota M, Yamada N, Aisaki $\mathrm{K}$, et al. Clinicopathological studies of odontoma in 47 patients. J Oral Sci 1999; 41: 173-6
Yazışma Adresi:

Nuray BAĞCI

Gazi Üniversitesi

Diş Hekimliği Fakültesi

Ağız Diş ve Çene Radyolojisi AD,

Ankara, Türkiye

E-mail : dtnuraybagci@gmail.com 